\title{
Medevac from a cruise ship of a patient with spontaneous coronary artery dissection who presented with epigastralgia
}

\author{
Jenny Garcia-Castaneda, Alfredo Harb-De la Rosa
}

Medical Department Carnival Cruise Lines, Miami, Florida, United States

\begin{abstract}
Spontaneous coronary artery dissection (SCAD) is a rare cause of acute coronary syndrome that has been associated with peripartum and postpartum periods. It results from the separation of the layers of the arterial wall of the coronary artery with the subsequent formation of a false lumen. We report a case of a 54-year-old female who presented to the cruise ship's medical facility complaining of epigastralgia and dizziness. Work up including an electrocardiography and cardiac profile was ordered. Results yielded a diagnosis of non-ST segment elevation myocardial infarction (NSTEMI). Treatment following American Heart Association recommendations including nitrates, clopidogrel and enoxaparin was given. After debarkation at sea and referral to a reference hospital, the patient was diagnosed with SCAD. Patient's outcome was favorable and she was discharged home a few days after, despite being managed as a NSTEMI.
\end{abstract}

(Int Marit Health 2014; 65, 3: 122-123)

Key words: spontaneous coronary artery dissection, epigastralgia, cardiac enzymes, ECG

\section{INTRODUCTION}

Spontaneous coronary artery dissection (SCAD) is a rare cause of acute coronary syndrome (ACS), however its recognition is increasing overtime. SCAD has been associated with peripartum and postpartum periods although its origin remains unknown [1]. Coronary artery dissection results from the separation of the layers of the arterial wall of the coronary artery with the subsequent formation of a false lumen. Thrombosis can develop as a consequence of a haemorrhage into the false lumen which may compress the true lumen causing myocardial ischaemia [2]. The mean age of presentation ranges from 30 to 45 years with female gender constituting $70 \%$ of the diagnosed cases [3]. Several cases of SCAD have been reported in association with connective tissue disorders, eosinophilic arteritis, contraception use, cocaine abuse, and repetitive chest trauma [4]. Retrospective analyses of large data-bases of coronary angiograms have shown a quite good survival rate after initial event despite of the dramatic starting presentation and high early mortality rate. Unsuccessful revascularisation procedures and myocardial infarction complications were the most common causes of high early mortality rate [5]. The optimal treatment strategy for SCAD remains unclear with percutaneous coronary intervention being unsuccessful in 35\% of cases and a conservative approach in stable, asymptomatic patients resulting in spontaneous resolution [6]. A large prospective study provided robust evidence that spontaneous healing of the vessel wall should be considered as part of the natural history of the disease. Therefore, initial conservative approach was recommended for all stable patients and revascularisation procedures were suggested for recurrence of ischaemia [7].

\section{CASE REPORT}

A 54-year-old female who came to the ship's medical facility complaining of epigastric discomfort/pain of recent onset, described as "a stone sensation inside". The patient 
Table 1. Cardiac enzymes

\begin{tabular}{llll}
\hline Test & $\begin{array}{l}\text { At } \\
\text { admission }\end{array}$ & 2 hours & 4 hours \\
& 0.16 & 1.99 & 2.63 \\
\hline Troponin I $[\mathrm{ng} / \mathrm{mL}]$ & 14.6 & 19.8
\end{tabular}

rated the pain as $6 / 10$ on a scale from 1 to 10 with 10 being the most intense pain. She denied any chest pain or pressure on her chest. Her symptoms started at rest and were associated with mild dizziness. She denied radiation of the epigastric discomfort as well as previous similar episodes. She tried 1 tablet of ranitidine without alleviation of the symptoms. The patient denied any remarkable medical or surgical history. Vital signs at admission: blood pressure 141/90 mm Hg, heart rate $79 \mathrm{bpm}$, respiratory rate 14 respirations per minute, peripheral oxygen saturation 96\%. Physical examination revealed an alert and oriented patient, with moist mucous membranes, equally reactive pupils, supple neck without jugular venous distention, rhythmic heart sounds without murmurs, normal S1 and S2 and clear lungs without aggregates. Abdomen, extremities and neurologic examination were unremarkable. The patient was admitted for evaluation. An electrocardiography (ECG) was initially ordered which revealed normal sinus rhythm without changes in the ST segment. Cardiac enzymes were ordered and results are shown in Table 1. A diagnosis of non-ST segment elevation myocardial infarction (NSTEMI) was considered. Management was started with oxygen, aspirin, sublingual nitroglycerine, clopidogrel, subcutaneous enoxaparin, metoprolol and maintenance IV fluids. During admission, the patient reported another episode of epigastric pain and an ECG was ordered immediately during the episode. No changes relative to admission ECG were noted. A drip of IV nitroglycerine was started which kept the patient without epigastralgia for the remaining time of the admission on board. The patient was subsequently Medevac via tender boat escorted by a ship's physician and referred to a hospital with available cath lab. Feedback from the hospital confirmed the diagnosis of SCAD. Also coronary angiography and placing of a stent was done. No further information regarding the intrahospital management provided to the patient was received. However, the patient sent us a written notification where she stated that she was feeling well after being discharged from the hospital.

\section{DISCUSSION}

It has been reported in the literature that SCAD predominates in females (70\%) which fits with our patient's gender; however, the age range reported in the literature (30-45) did not correspond with our patient's age of presentation. SCAD may be a difficult if not impossible condition to diagnose on a cruise ship medical facility, mainly because diagnostic tools i.e. (computed tomography angiogram, cath lab) are, of course, not available. Nevertheless, SCAD must be considered as part of the differential diagnosis in patients with ACS. Our patient's chief complaint was epigastric pain/discomfort associated with mild dizziness without any important haemodynamic alteration. As a rule, emergency physicians always include an ECG in the work up of epigastric pain. In our case, the ECG was normal. The decision of continuing the investigation of a possible ACS or ruling it out based on a normal ECG, became a critical point in the management of this patient. As shown in Table 1, serial elevation of cardiac enzymes yielded a diagnosis of ACS. The most likely diagnosis among the differentials of ACS was NSTEMI according to the ECG and cardiac profile findings. Treatment was established focusing on that diagnosis. Since the patient's actual diagnosis ended up being SCAD, this brings at least one question to mind. Was it appropriate to administer full doses of aspirin, clopidogrel and enoxaparin to a SCAD patient when bleeding might be stemming from the dissection itself? This case stands as an example of how challenging is to diagnose and treat a patient with SCAD in the setting of a cruise ship medical centre.

\section{REFERENCES}

1. Tokura M, Taquichi I, Kageyama $\mathrm{M}$ et al. Clinical features of spontaneous coronary artery dissection. J Cardiol 2014; 63: 119-122.

2. Vrints CJ. Spontaneous coronary artery dissection. Heart 2010; 96: 801-808.

3. Sivam S, Yozghatlian V, Dentice R et al. Spontaneous coronary artery dissection associated with coughing. J Cyst Fibros 2014; 13: 235-237.

4. Junpaparp P, Romero-Corral A, Lee SY et al. Spontaneous coronary artery dissection: a rare cause of acute coronary syndrome. BMJ Case Rep 2014; 2014.

5. Putnikovic B, llić I, Panić $M$ et al. Spontaneous coronary artery dissection - rare but challenging. Vojnosanit Pregl 2014; 71: 311-316.

6. Mathur M, Huda N, Cohen H, Parris T. Spontaneous right coronary artery dissection: a case of spontaneous resolution. J Am Coll Cardiol Cardiovasc Interv 2014; 7: e9-e10.

7. Alfonso F, Perez-Vizcayno MJ, Hernandez R et al. Spontaneous coronary artery dissection: long-term follow-up of a large series of patients prospectively managed with a "conservative" therapeutic strategy. J Am Coll Cardiol Cardiovasc Interv 2012; 5: 1062-1070. 